\title{
GMR
}

\section{Cloning and characterization of ChiMYB in Chrysanthemum indicum with an emphasis on salinity stress tolerance}

\author{
M. He ${ }^{1}$, H. Wang1, Y. Z. Liu ${ }^{2}$, W.J. Gao ${ }^{1}$, Y.H. Gao ${ }^{2}$, F. Wang ${ }^{1}$ and Y.W. Zhou ${ }^{1}$ \\ ${ }^{1}$ College of Landscape Architecture, Northeast Forestry University, \\ Harbin, China \\ ${ }^{2}$ College of Landscape Architecture, Beijing Forestry University, \\ Beijing, China \\ Corresponding author: Y.W. Zhou \\ E-mail: zhouyunwei@hotmail.com \\ Genet. Mol. Res. 15 (3): gmr.15038985 \\ Received July 21, 2016 \\ Accepted August 1, 2016 \\ Published September 23, 2016 \\ DOI http://dx.doi.org/10.4238/gmr.15038985
}

Copyright (C) 2016 The Authors. This is an open-access article distributed under the terms of the Creative Commons Attribution ShareAlike (CC BY-SA) 4.0 License.

\begin{abstract}
. v-myb avianmyeloblastosis viral oncogene homolog (MYB) transcription factors are key regulators of stress responsive gene expression in plants. In this study, the MYB gene, ChiMYB (GenBank accession No. KT948997), was isolated from Chrysanthemum indicum, and was functionally characterized with an emphasis on salinity stress tolerance. The full ChiMYB cDNA sequence (948 bp) encoded a typical R2R3 MYB transcription factor that contained 315 amino acid residues and two MYB domains. The temporal expression pattern of ChiMYB was noted in C. indicum, and the highest level was detected in the roots, followed by leaves and stems. ChiMYB expression was induced by $\mathrm{NaCl}$ treatments, and transient expression of the fusion of ChiMYB and green fluorescent protein $(G F P)$ indicated that the protein was targeted to the nuclei of onion epidermal cells. Arabidopsis plants
\end{abstract}


overexpressing ChiMYB displayed improved tolerance to drought and salt stress. When under salt stress conditions, transgenic Arabidopsis plants had higher survival rates than non-transgenic wild-type plants. Chlorophyll content, intercellular $\mathrm{CO}_{2}$ concentration, photosynthetic rate, and stomatal conductance were higher in the transgenic Arabidopsis plants than in non-transgenic control plants. Further investigation revealed that $C h i M Y B$ was able to regulate the expression of $R D 29 A$, $R A B 18, C O R 15, A B I 1$, and $A B A$ genes, which are involved in salt stress signaling pathways. Our findings demonstrated that ChiMYB is essential for plant responses to salt stress, and it may have great potential for the improvement of salt tolerance in crops.

Key words: Chrysanthemum indicum; Arabidopsis thaliana; ChiMYB; Salt stress; Photosynthesis; qRT-PCR

\section{INTRODUCTION}

Salinity can significantly and negatively affect plant growth, development, and productivity. Serious salt stress can cause the substantial loss of crop production, and it may even lead plant death. Several characteristics of salt-stressed plants lead to the alteration of the photosynthetic parameters of plant leaves (Sudhir and Sistla, 2004), including damaged cytochrome systems, reduced chlorophyll content, closed stomata, decreased $\mathrm{CO}_{2}$ concentrations, and reduced photosynthetic pigment content (Hoshida et al., 2000; Jin, 2002; Jiang et al., 2008; Shu et al., 2012a). These alterations are often associated with the degree and duration of salt stress (Lakshmi et al., 1996; Misra et al., 1997). Therefore, it has become an urgent need to genetically develop plants with significantly improved tolerance to salinity.

Transcription factors are proteins with special structures and functions that regulate the expression of other genes, and they are essential to plant defensive responses to biotic and abiotic stress (Liu et al., 2008). To date, numerous transcription factors from different families, including v-myb avianmyeloblastosis viral oncogene homolog (MYB), ethylene resposive factor (ERF), basic region/leucine zipper motif (bZIP), and WRKY, were found to be involved in the regulation of stress responses (Schwechheimer et al., 1998; Singh et al., 2002). MYB proteins are one of the largest families of plant transcription factors (Tang and Chen, 2014). Moreover, most MYB members belong to the R2R3-MYB type (Shu et al., 2012b), and they are involved in the regulation of plant secondary metabolism and responses to different stresses (Uimari and Strommer, 1997; Hoeren et al., 1998; Lea et al., 2007; Du et al., 2008). Different members of the MYB family may have varying tempo-spatial expression patterns, and they may function differently in plants (reference needed). For instance, AtMYB2 regulates responses to dehydration and salt stresses in Arabidopsis (Martin and Paz-Ares, 1997). Enhanced expression levels of OsMYB4 in rice significantly improve the tolerance of transgenic plants to drought, salinity, and UV radiation (Vannini et al., 2004). In addition to drought-induced expression, BcMYB1 from Boea crassifolia also responds to different stresses such as polyethylene glycol (PEG), salinity, and low temperatures (Chen et al., 2005). Furthermore, transcription factors generally contain a short-span nuclear localization signal (NLS) that is often rich in arginine and lysine residues, and it also regulates the entry process of the transcription factor into cell nuclei.

Genetics and Molecular Research 15 (3): gmr.15038985 
Since the promoters of different stress-induced genes often contain the same cis acting elements, these genes can be regulated by the same transcription factors (Liu et al., 2008). A transcription regulator might possibly modulate a set of functional genes responsible for plant tolerance to the stressor, which consequently improves plant stress tolerance. Thus, manipulation of transcription factor expression has great potential for the improvement of plant resistance to stress.

Chrysanthemum indicum is a perennial herbaceous species that is spreading widely in China, and it exhibits variably colored flowers and tolerance to different stresses. In this study, the R2R3-MYB gene, ChiMYB, was cloned and functionally characterized for a better understanding of its role in plant responses to salt stress and its potential applications to the improvement of stress resistance in crops.

\section{MATERIAL AND METHODS}

\section{Plants and salt stress}

C. indicum seeds were introduced to the nursery of the Northeast Forestry University from Benxi City $\left(121^{\circ} 21^{\prime} \mathrm{E}, 41^{\circ} 22^{\prime} \mathrm{N}\right)$, Liaoning Province, China. The cutting plants were obtained in November 2014, and were potted (diameter $12 \mathrm{~cm}$ ) in mixed media (garden soil:vermiculite, 1:1) without additional fertilizer in March 2015. The plants were maintained in a greenhouse under standard growing conditions. Young plants were watered daily and fertilized weekly using half strength Hoagland's nutrient solution. Clipped leaves were frozen in liquid nitrogen and cryopreserved at $-80^{\circ} \mathrm{C}$ for RNA extraction.

Arabidopsis thaliana ecotype Columbia (Col-0) plants were cultivated in pots filled with a mixture of perlite:vermiculite:soilrite (v:v:v, 1:1:1) in a growth chamber with $16 \mathrm{~h}$ of light $(60-100 \mathrm{~lx})$ at $23^{\circ}$ and $18^{\circ} \mathrm{C}$ for day and night, respectively. Relative humidity in the chamber was maintained at $60 \%$, and the plants were watered every four days until floral dip transformation occurred.

For the functional characterization of ChiMYB, non-transformed wild-type plants and transgenic Arabidopsis plants that overexpressed ChiMYB were treated with $250 \mathrm{mM} \mathrm{NaCl}$. The rosette leaves were excised at $0,1,3,6,12$, and $24 \mathrm{~h}$ after the salt treatment, and were then stored at $-80^{\circ} \mathrm{C}$ until use. Twenty-day-old non-transgenic and transgenic plants were used for RNA extraction and gene expression analyses.

\section{cDNA and gDNA cloning}

Total RNA was isolated from C. indicum leaves using the RNAiso reagent (TIANGEN, Beijing, China), and cDNA was then synthesized using a Revert AidTM First Strand reverse transcription kit (TOYOBO Japan). Based on conserved sequence information from homologous MYB genes in Arabidopsis, alfalfa, and other species in the GenBank database at the National Center for Biotechnology Information (NCBI), degenerate primers (MYB-F and MYB-R; Table 1) were designed using Primer5.0 software. The polymerase chain reaction (PCR) products were purified and cloned into the pMD18-T vector (TaKaRa Japan), which was introduced into E. coli DH5 $\alpha$. The inserts in plasmids isolated from positive clones after PCR screening were sequenced. BLAST analyses, using the isolated MYB gene as query against the GenBank database, were performed to obtain further annotation. The FullMYB-F

Genetics and Molecular Research 15 (3): gmr.15038985 
and FullMYB-R primers (Table 1) were designed using Primer5.0, and were then used to clone the full-length transcript. The cloned gene was then sequenced for confirmation.

\begin{tabular}{|c|c|}
\hline Primer & Sequences $\left(5^{\prime}-3^{\prime}\right)$ \\
\hline \multirow[t]{2}{*}{$M Y B$} & F: GTCCAGACGAGGACGAAATGT \\
\hline & R: GTCTTATCGGCATCACATTCG \\
\hline \multirow[t]{2}{*}{ FullMYB } & F: САТССТСТСАТTTTCTTTCCAA \\
\hline & R: CTACCCTCATCATATCCCATCTC \\
\hline \multirow[t]{2}{*}{ MYB-1301 } & F: GGATCCATGGCTAGTGTTTCAAA \\
\hline & R: CGAGCTCCTAATCTATTTTACTAATCCC \\
\hline \multirow[t]{2}{*}{$A t U B Q$} & F: GGTTCGTACCTTTGTCCAAGCA \\
\hline & R: CCTTCGTTAAACCAAGCTCAGTATC \\
\hline \multirow[t]{2}{*}{ RD22 } & F: ACTTGGTAAATATCACGTCAGGGCT \\
\hline & R: CTGAGGTGTTCTTGTGGCATACC \\
\hline \multirow[t]{2}{*}{$R D 29 A$} & F: GATAACGTTGGAGGAAGAGTCGG \\
\hline & R: TCCTGATTCACCTGGAAATTTCG \\
\hline \multirow[t]{2}{*}{$R A B 18$} & F: GCAGTATGACGAGTACGGAAATCC \\
\hline & R: CCTTGTCCATCATCCGAGCTAGA \\
\hline \multirow[t]{2}{*}{ COR47 } & F: TGACGGATAAAACAAAAGAGG \\
\hline & R: GACCCTACTTTGTGGCATCCTT \\
\hline \multirow[t]{2}{*}{$A B A 1$} & F: GCTATGAAGGTGATCTGCTTGTGG \\
\hline & R: TTCATACCATTTGGAGCATCAGC \\
\hline \multirow[t]{2}{*}{$A B I 1$} & F: AGCTGCTGATATAGTCGTCGTTGATA \\
\hline & R: GAGGATCAAACCGACCATCTAACA \\
\hline \multirow[t]{2}{*}{ HABI } & F: TTCTCGCCATGTCTAGGTCCAT \\
\hline & R: TTCGCAGACTTCTTGGTTGTTC \\
\hline
\end{tabular}

\section{Phylogenetic analysis of ChiMYB}

The open reading frame (ORF) of ChiMYB was identified using the ORFfinder program, and the structures of the conserved domains were examined using NCBI BLAST analyses and DNAMAN software. Phylogenetic analyses were performed with MYB protein sequences from other plant species.

\section{Transgenic $A$. thaliana}

The ChiMYB coding sequence, with additional BamHI and $\mathrm{SacI}$ restriction sites at the 5' and 3' ends, respectively, was amplified using the MYB-1301-F/MYB-1301-R primer pair (Table 1). The amplified product was then purified and cloned into MYB-PMD18-T, which is a pMD18-T vector (TaKaRa). The insert was retrieved using BamHI and SacI, and was inserted into a pCAMBIA1301 vector that was digested with the same enzyme pair. The construct was then introduced into Agrobacterium tumefaciens EHA105 for Arabidopsis floral dip transformation (Clough and Bent, 1998). The seeds from Agrobacterium-infected Arabidopsis plants were sown into half-strength Murashige and Skoog (MS) medium that contained $50 \mathrm{mg} / \mathrm{L}$ of hygromycin for transgenic plant screening. Surviving plants were rescreened and self-pollinated, and the process was repeated until homozygous T4 transgenic Arabidopsis lines were obtained. Total RNA was extracted from the young leaves of transgenic and non-transgenic plants using the TRIZOL extraction method, and transgene expression was quantified using $M Y B$-specific primers.

Genetics and Molecular Research 15 (3): gmr.15038985 


\section{Subcellular localization of ChiMYB}

The complete ChiMYB ORF, with the addition of $X b a \mathrm{I}$ and SpeI restriction sites at the ends, was amplified via PCR using primers MYB-121-F/R (Table 1). The PCR product (confirmed via sequencing) was digested with $X b a \mathrm{I}$ and SpeI, and was then inserted into pBI121 to create a fusion construct (pBI121-ChiMYB-GFP). The same construct, without ChiMYB (pBI121-GFP), was used as a control. Onion epidermal cells were bombarded with both the fusion and control vectors using a GJ-1000 Gene Gun System (Scientz, China). After $24 \mathrm{~h}$ at $25^{\circ} \mathrm{C}$ in darkness, the fluorescent signals from GFP and ChiMYB-GFP fusion samples were observed using a confocal laser scanning microscope (Zeiss LSM510, Germany).

\section{Salt stress tolerance assay of Arabidopsis plants expressing ChiMYB}

Germination assays, comprised of 100 transgenic or non-transgenic seeds, were conducted on $1 / 2$ MS media containing $0,50,100,150$, or $200 \mathrm{mM} \mathrm{NaCl}$, and each assay was run in triplicate. Water-imbibed seeds were kept in the dark for $48 \mathrm{~h}$ at $4^{\circ} \mathrm{C}$, and seeds were then germinated in a growth chamber under normal growth conditions for 10 days. The germination rates of seeds (with $2 \mathrm{~mm}$ radicles) were presented as the percentage of the germinated seeds over the total number of seeds planted.

Regarding the salinity tolerance test, three-week-old non-transgenic Col-0 and overexpressed ChiMYB Arabidopsis plants were cultivated in pots filled with a mixture of perlite:vermiculite:soilrite (v:v:v, 1:1:1) in a growth chamber. Plants were exposed to different levels of $\mathrm{NaCl}(0,50,100,150$, and $200 \mathrm{mM})$, and were watered for 12 days at four-day intervals. Survival rates of ChiMYB transgenic and non-transgenic plants were obtained and statistically analyzed.

\section{Determination of chlorophyll content}

The chlorophyll $a(\operatorname{chl} a)$, chlorophyll $b(\operatorname{chl} b)$, total chlorophyll, and carotenoid contents in salt-stressed ChiMYB-expressing transgenic and non-transgenic Arabidopsis plants were determined using the Lichtenhaler and Wellburn (Lichtenhaler and Wellburn, 1983) method.

\section{Determination of the net photosynthetic rate, stomatal conductance, and intercellular $\mathrm{CO}_{2}$}

The net photosynthetic rate $(\mathrm{Pn})$, stomatal conductance $(\mathrm{Gs})$, and intercellular $\mathrm{CO}_{2}$ concentration $(\mathrm{Ci}$ ) of salt-treated Col-0 and ChiMYB-expressing transgenic and non-transgenic Arabidopsis plants were measured using a Licor-6400 (Ecotek Technology, United States) at $23^{\circ} \mathrm{C}$ with $50 \%$ relative humidity and a light intensity of $300 \mathrm{~lx}$. Leaf area was measured and calculated using a scanner (YMJ, China) and Leaf Data software, and each treatment was repeated at least four times.

\section{Quantitative reverse transcription PCR (qRT-PCR)}

To determine the expression patterns of ChiMYB in C. indicum under salt stress 
conditions, total RNA was isolated from fresh leaves (homogenized in liquid nitrogen) using the TRNzol Universal Reagent (TIANGEN), and genomic DNA contamination was eliminated using DNaseI. cDNA synthesis was conducted using random primers and a reverse transcription kit (TOYOBO, Japan). Quantitative PCR (qPCR) was performed using SYBR Green I (TOYOBO, Japan) and a LightCycler ${ }^{\circledR} 96$ Real-Time PCR System (Roche Diagnostics Ltd., Shanghai, PRC). The qRT-PCR mixture $(20 \mu \mathrm{L})$ contained $10 \mu \mathrm{L}$ SYBR Green PCR master mix, $0.4 \mu \mathrm{L}$ of each gene-specific primer (Table 1), and $0.5 \mathrm{ng}$ cDNA. The following qPCR protocol was used: $95^{\circ} \mathrm{C}$ for $60 \mathrm{~s} ; 40$ cycles of $95^{\circ} \mathrm{C}$ for $15 \mathrm{~s} ; 60^{\circ} \mathrm{C}$ at $15 \mathrm{~s}$; and $72^{\circ} \mathrm{C}$ for $45 \mathrm{~s}$. The data are represented by the means \pm SD of three replicates. The reference Arabidopsis gene $A t U B Q$ (NM_116771.5) was used for the normalization of ChiMYB expression via the $2^{-\Delta \Delta \mathrm{Ct}}$ method, where $\Delta \Delta \mathrm{Ct}=(\mathrm{Ct}$ of target gene - $\mathrm{Ct}$ of reference gene AtUBQ $)$ Time $_{\mathrm{x}}$ - (Ct of target gene - Ct of reference gene $A t U B Q$ ) Time ${ }_{0}$ (Livak and Schmittgen, 2001). For the Arabidopsis stress-responsive gene expression analyses, RNA was extracted from non-transgenic and ChiMYB-expressing plants, and cDNA was synthesized. Transcripts of the stress-responsive genes $R D 22, R D 29 A, R A B 18, C O R 15, A B A 1, A B I 1$, and $H A B 1$ were quantified using gene-specific primers (Table 1). The qPCR protocol for Arabidopsis gene expression was identical to that used for ChiMYB expression, with the exception of different gene-specific primers.

\section{RESULTS}

\section{Analysis of R2R3-MYB sequences from $C$. indicum}

In this study, ChiMYB (an MYB gene) was isolated from C. indicum, and it contained a full-length ORF (948 bp) that encoded a protein with 315 amino acid residues (GenBank accession No. KT948997). The encoded protein contained two MYB domains, and it functioned as a typical R2R3MYB transcription factor. The sequence alignment analysis indicated that ChiMYB shared 95\% similarity with chrysanthemum MYB2 (AE027498.1), 79\% with tomato MYB44 (XP003611666.1), and 76\% with an alfalfa homologue (XP003611666.1). Multiple sequence alignment of the ChiMYB protein was performed against 13 MYB homologues

from different plant species using DNAMAN software (Figure 1), and the results revealed conserved domains across all of the tested MYB proteins.

The reconstructed phylogenetic tree suggested that the tested MYB proteins could be divided into four groups (Figure 2). Group 1 included Chrysanthemum CmMYB (Chrysanthemum x morifolium, AE027498.1), castor oil RcMYB (Ricinus communis, XP002524926.1), and a Chrysanthemum MYB transcription factor, which are all associated with hormonal responses (Shan et al., 2012). Group 2 contained samples from Epimedium EsMYB (Epimedium sagittatum, AFH03065.1), CjMYB (Coptis japonica, BAJ40867.1), grape VvMYB (Vitis vinifera, XP002285015.1), and tobacco NtMYB (Nicotiana tabacum, BAC53938.1), which were all involved in the metabolism of flavonoids, abiotic stress, and embryonic development (Masaharu et al., 2003; Huang et al., 2013); Group 3 included soybean MYB (Glycine max, NP001235142.1), chickpea CaMYB (Cicer arietinum, XP004511885.1), alfalfa MtMYB (Medicago truncatula, XP003611666.1), and cucumber CsMYB (Cucumis sativus, XP004151866.1), which are associated with abiotic stress. Group 4 included Arabidopsis AtMYB30 (Arabidopsis thaliana, CAA90809.1), tomato SiMYB (Solanum lycopersicum, XP004231882), and orange CSMYB (Citrus sinensis, ABQ10816.1), which are

Genetics and Molecular Research 15 (3): gmr.15038985 
involved in the regulation of pigment metabolism (Kirik et al., 1998; Luo et al., 2008; Yang et al., 2008; Antonietta and Paola, 2010). Moreover, the phylogenetic analysis showed that the ChiMYB protein was closely related to the Chrysanthemum samples, which suggests that it might function similarly to other Chrysanthemum MYB transcription factors (Figure 2).

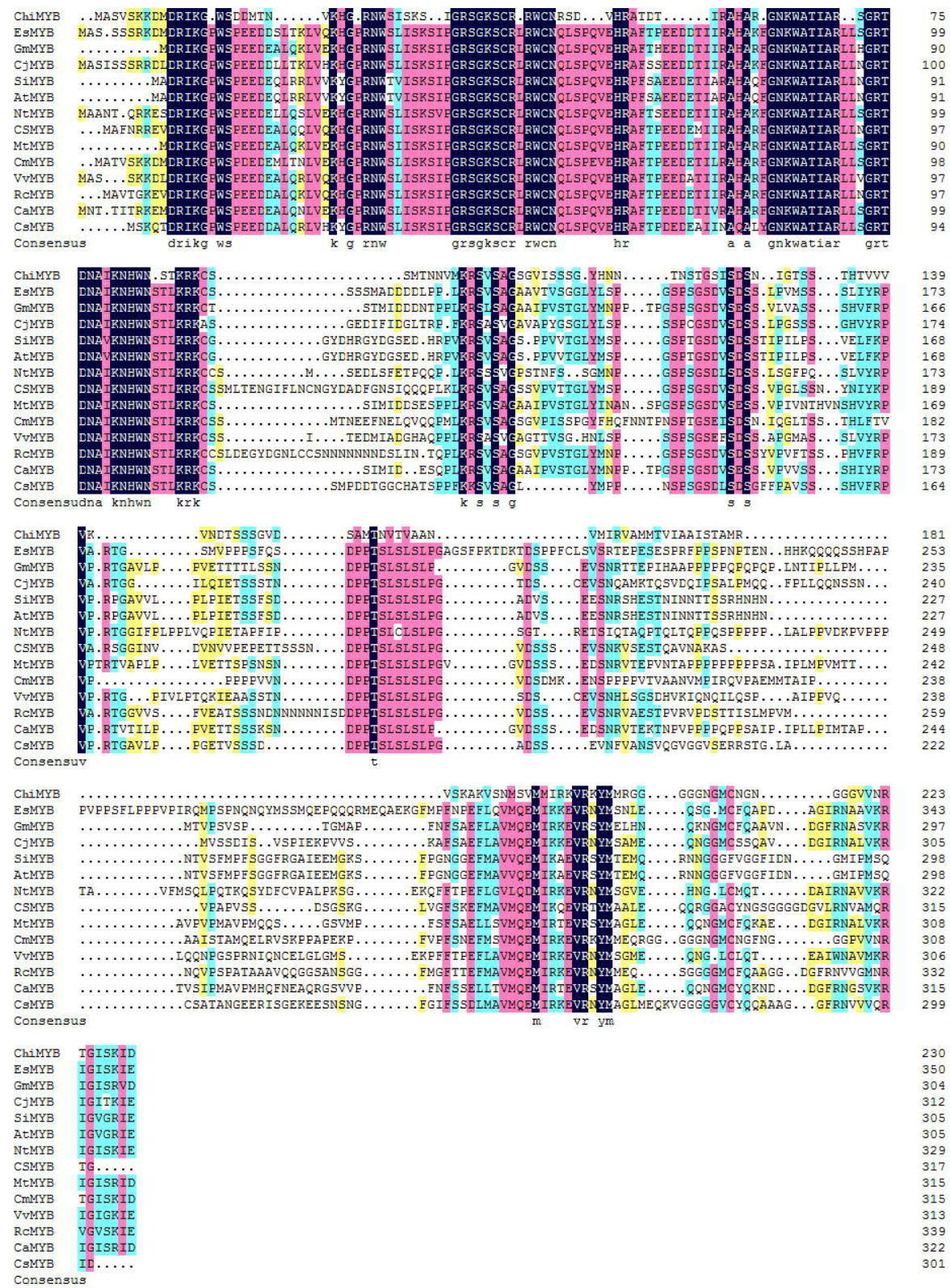

Figure 1. Alignment of MYB proteins from different plant species. The conserved SANT DNA-binding domains are highlighted.

Genetics and Molecular Research 15 (3): gmr.15038985 


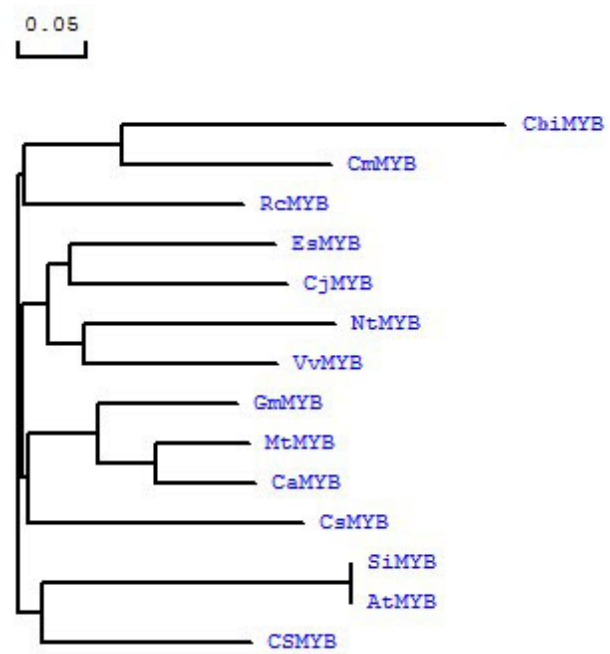

Figure 2. Phylogeny of ChiMYB and samples from other species.

To confirm the subcellular location of ChiMYB, 35S:ChiMYB-GFP and 35S:GFP were constructed and introduced into onion epidermal cells. Confocal microscopy observations revealed that the ChiMYB-GFP fusion was localized to the nucleus. However, GFP (alone) was distributed throughout the cell. Therefore, these results indicated that ChiMYB was localized to the nucleus, which is consistent with its predicted function as a transcription factor (Figure 3).

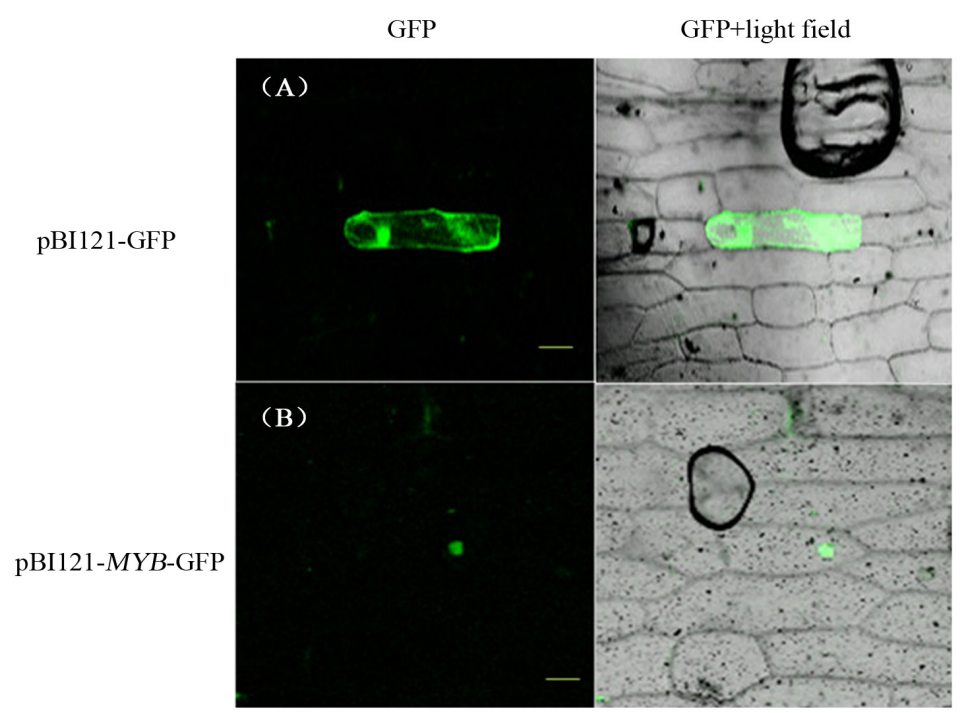

Figure 3. ChiMYB subcellular localization revealed by GFP fusion. The constructs carrying GFP alone (A) or ChiMYB-GFP were both driven by the CaMV 35S promoter, which was introduced into onion epidermal cells using a gene gun (B). The transformed samples were maintained at $22^{\circ} \mathrm{C}$ for $24 \mathrm{~h}$, and were observed under a confocal microscope. Scale bars $=50 \mu \mathrm{m}$.

Genetics and Molecular Research 15 (3): gmr.15038985 


\section{Alteration of seed germination and plant tolerance in transgenic plants under salt stress}

After hygromycin screening of $\mathrm{T}_{0}$ generation seeds, $\mathrm{T}_{1}$ seedlings of the three independent lines (MYB-1, $M Y B-2$, and $M Y B-3)$ were obtained. Continuous selfing was conducted until $\mathrm{T}_{3}$ homozygotes of the three lines were acquired (Figure 4A). The results of GUS staining, which was used to screen for transgenic A. thaliana, are shown in Figure 4B.
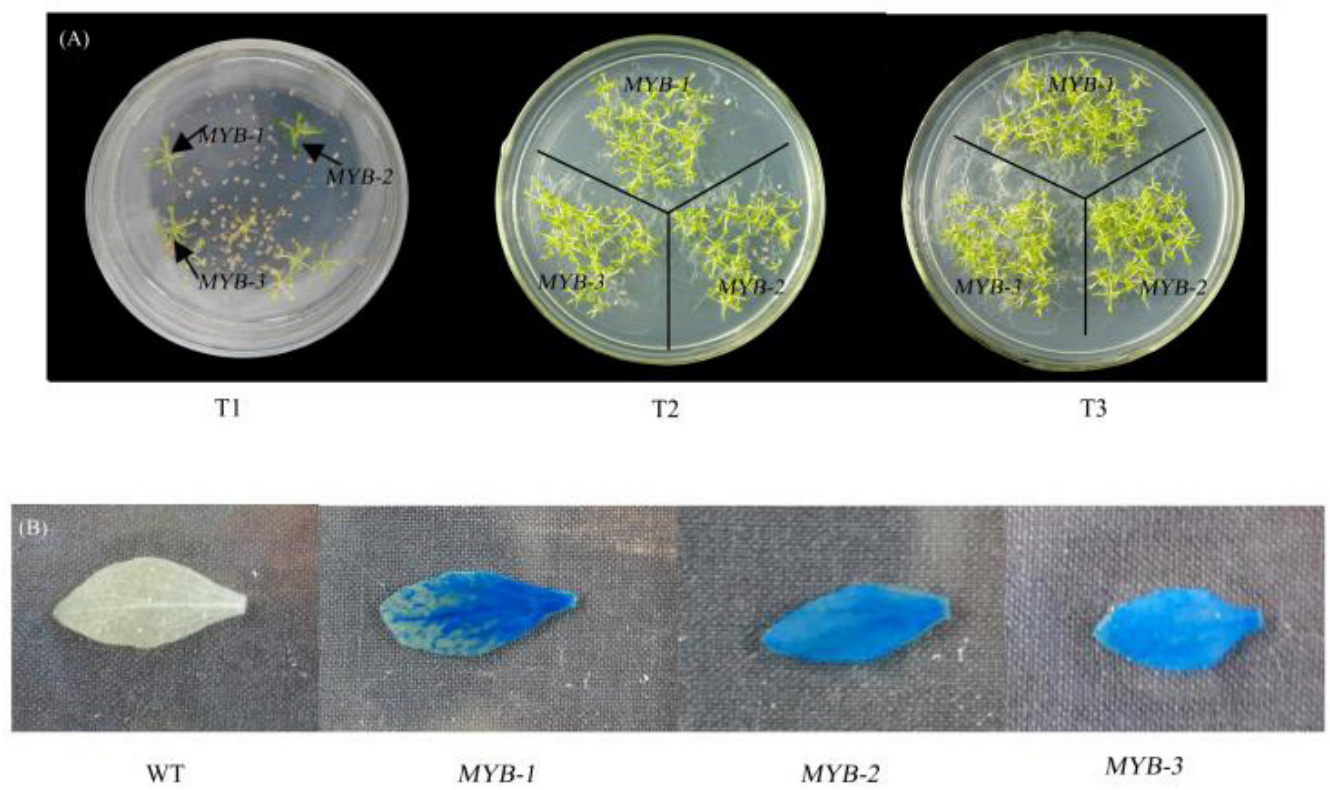

Figure 4. Positive transgenic Arabidopsis plants in selection media containing hygromycin (A). GUS stain screening for transgenic $A$. thaliana $(\mathbf{B})$.

When salt was not added to the medium, the transgenic Arabidopsis plants germinated normally. Furthermore, the germination rate of transgenic Arabidopsis seeds in the presence of $50 \mathrm{mM} \mathrm{NaCl}$ was over $90 \%$, and similar results were also found for Col-0 seeds. However, when the salt concentration was increased to $100 \mathrm{mM}$, the transgenic lines still maintained high germination rates $(91.8,91.3$, and $93.3 \%$ for $M Y B-1, M Y B-2$, and $M Y B-3$, respectively). In contrast, the germination rate of Col-0 seeds decreased to $55.6 \%$. When the $\mathrm{NaCl}$ concentration was further increased to 150 or $200 \mathrm{mM}$, the average germination rates of the three transgenic seed lines were 48 and $36 \%$, respectively. However, that of the Col-0 seeds at 150 or $200 \mathrm{mM}$ decreased to 18 and $9 \%$, respectively (Figure 5A). Moreover, as the $\mathrm{NaCl}$ concentration increased, the survival rates of transgenic and non-transgenic Col- 0 plants gradually decreased. The leaves of transgenic Arabidopsis plants wilted slowly, whereas Col0 leaves wilted rapidly. At a concentration of $250 \mathrm{mM} \mathrm{NaCl}$, the survival rates of the three transgenic lines (MYB-1, MYB-2, and $M Y B-3)$ were 72,83 , and 79\%, respectively. In contrast, that of Col-0 plants was only $30.1 \%$ (Figure $5 \mathrm{~B}$ ). 

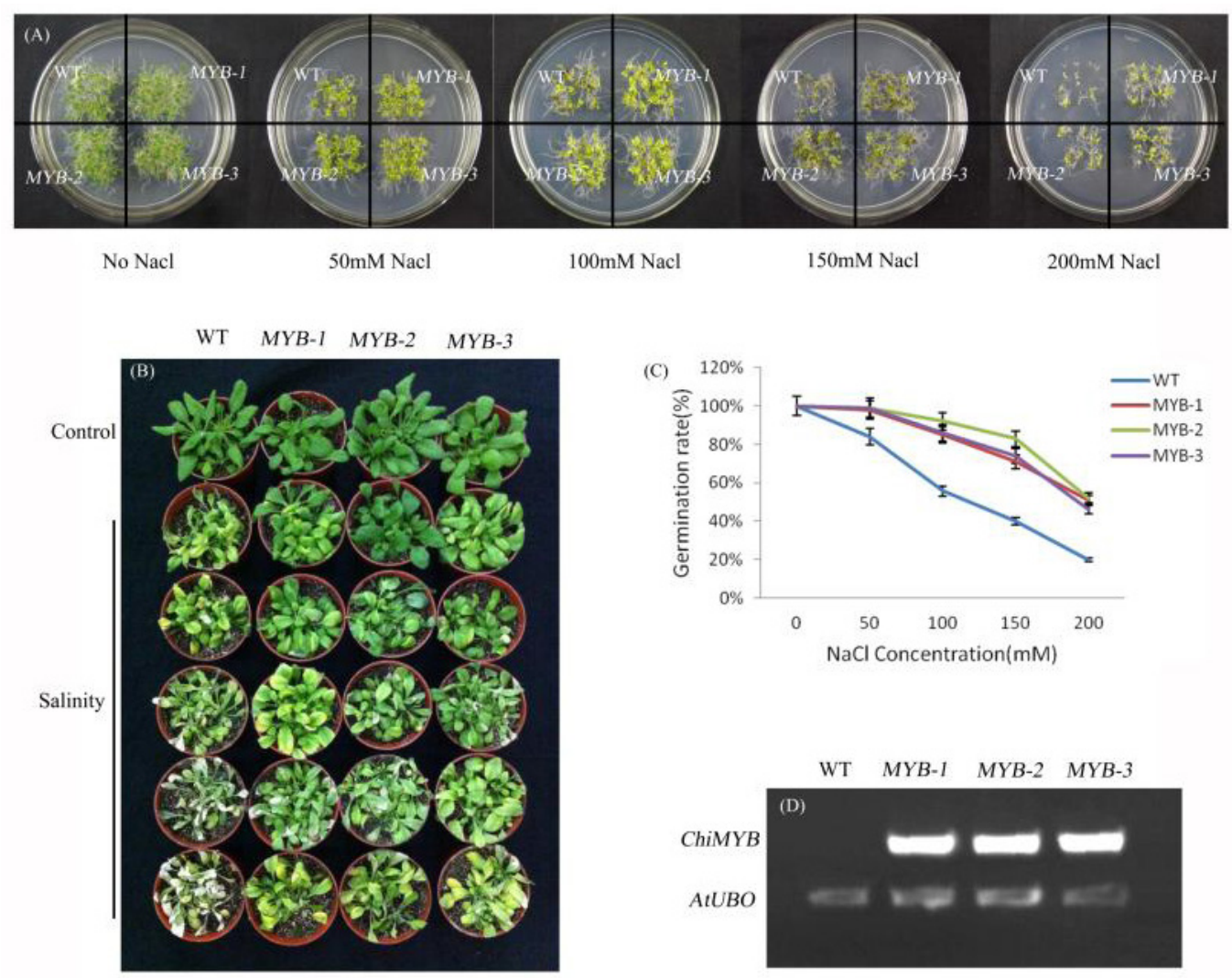

Figure 5. Seed germination and growth of overexpressed ChiMYB in Arabidopsis under different levels of salt stress. Seed germination under different salt stress conditions (A and $\mathbf{C})$, plant tolerance at different salt concentrations (B), PCR-detected presence of transgenes and internal reference genes (AtUBQ) in transgenic plants (D).

\section{Chlorophyll content in transgenic Arabidopsis plants}

The ratio of chla to chlb in both transgenic and non-transgenic Arabidopsis plants decreased as the $\mathrm{NaCl}$ concentration increased. Compared with that in transgenic Arabidopsis plants, the ratio in non-transgenic controls was greatly reduced. When the $\mathrm{NaCl}$ concentration was 200 or $250 \mathrm{mM}$, the chla/chlb values of wild-type Arabidopsis plants decreased to the lowest values, while those of transgenic Arabidopsis plants slightly declined. These results suggested improved salt tolerance in transgenic plants (Figure 6A).

As the salt stress conditions intensified, the carotenoid/chlorophyll ratios (Car/ Chl) of the transgenic Arabidopsis plants gradually increased until the salt concentration reached $150 \mathrm{mM}$ and then declined. However, the $\mathrm{Car} / \mathrm{Chl}$ values of non-transgenic Col0 plants peaked at $50 \mathrm{mM} \mathrm{NaCl}$ before rapidly declining. These results demonstrated that transgenic Arabidopsis plants were more tolerant to salt stress conditions than nontransgenic plants $(\mathrm{P}<0.05)$ (Figure 6B). 


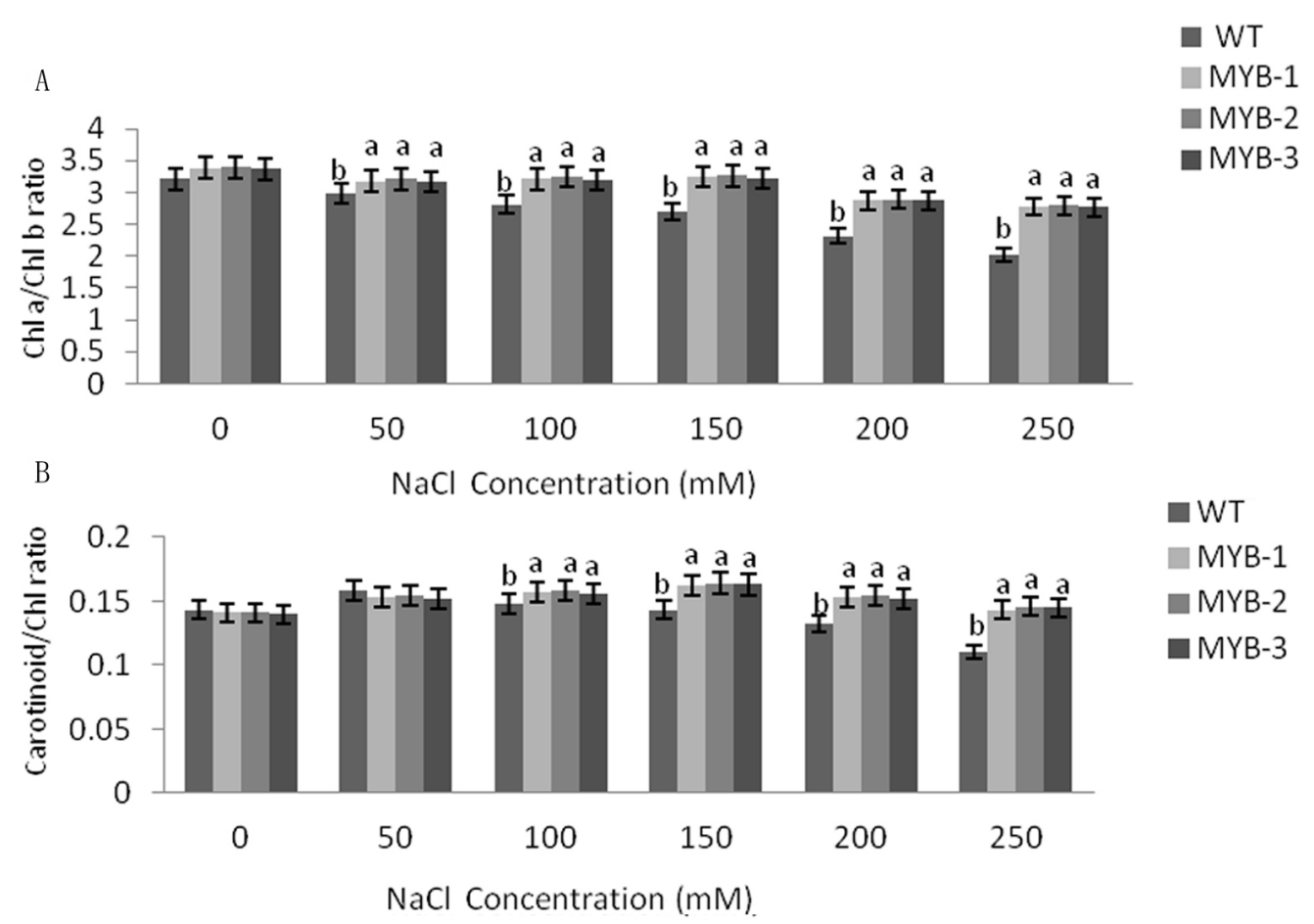

Figure 6. Changes in the ratios of chlorophyll a/b (A) and carotenoid/chlorophyll (B) in transgenic and nontransgenic Arabidopsis plants under different salt concentrations.

\section{Photosynthetic rates of transgenic Arabidopsis plants}

As the $\mathrm{NaCl}$ concentration increased, $\mathrm{Gs}, \mathrm{Ci}$, and $\mathrm{Pn}$ values decreased in Col-0 and the three transgenic Arabidopsis lines (Figure 7). Compared to the transgenic Arabidopsis plants, a greater reduction in the above parameters occurred in non-transgenic plants (Col-0). When $\mathrm{NaCl}$ concentration was $50 \mathrm{mM}$, transgenic Arabidopsis plants maintained Pn values compared to those not under salt stress conditions. However, when the $\mathrm{NaCl}$ concentration increased to over $100 \mathrm{mM}$, the Pn values of the three transgenic lines declined, but a sharper decline in Pn occurred in non-transgenic plants. At a concentration of $250 \mathrm{mM} \mathrm{NaCl}$, the Gs value of Col-0 decreased significantly. However, at concentrations up to $250 \mathrm{mM} \mathrm{NaCl}$, the Gs values of the three transgenic Arabidopsis lines did not change significantly. The $\mathrm{Ci}$ concentrations of Col- 0 and the three transgenic Arabidopsis plants decreased with increasing $\mathrm{NaCl}$ concentrations. For instance, at a concentration of $100 \mathrm{mM} \mathrm{NaCl}$, the Ci rates of both transgenic and non-transgenic plants were significantly lower, and they continued to decline with increasing $\mathrm{NaCl}$ concentrations. However, a faster decline in $\mathrm{Ci}$ values was observed in Col-0 than for the transgenic strains.

\section{Differential ChiMYB expression in transgenic Arabidopsis plants}

Transcript quantification results indicated that the expression levels of ChiMYB 
differed in the tested plant organs. The highest transcription was found in the stems, and the lowest was found in the roots (Figure 8).
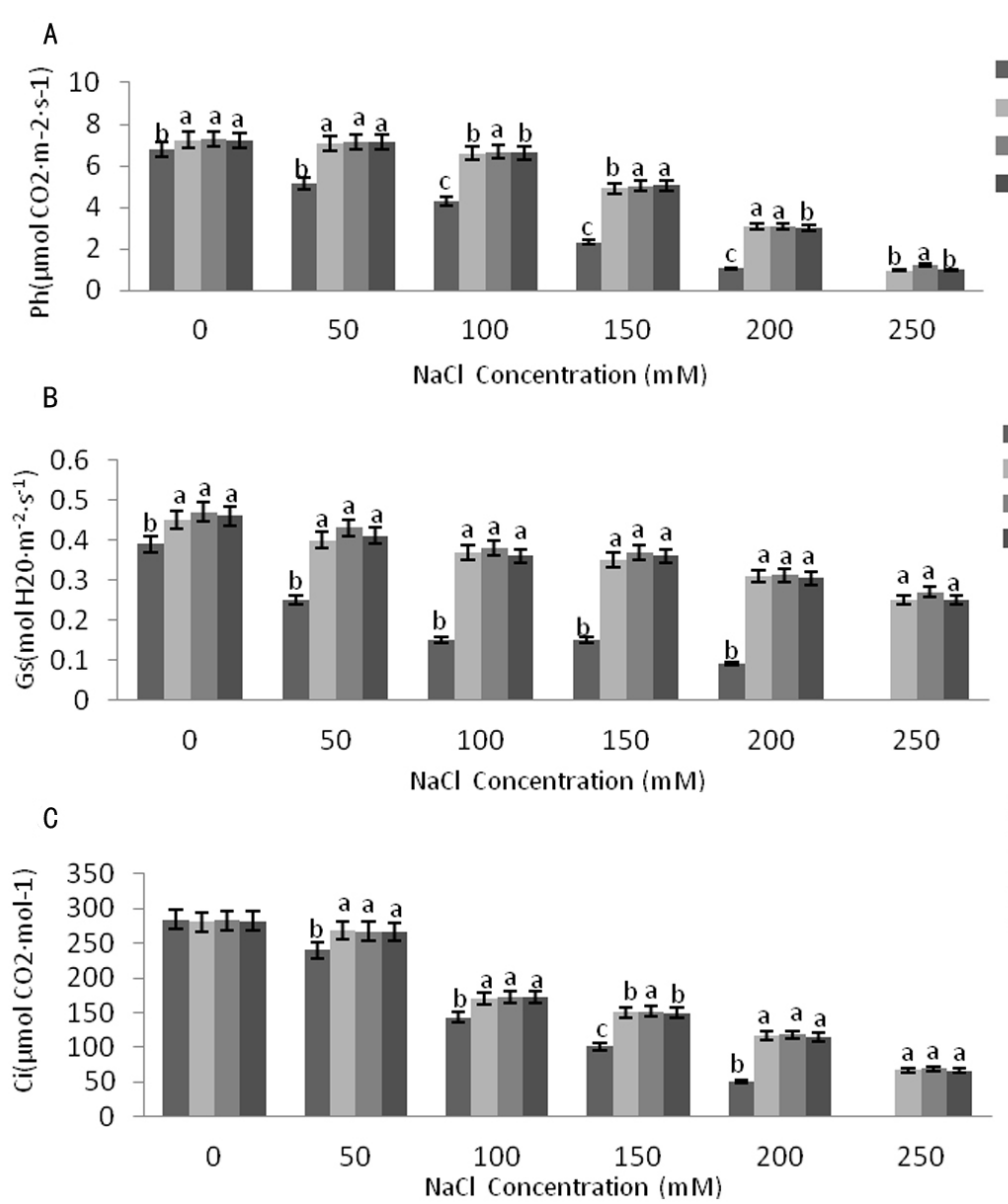

Figure 7. Changes in photosynthetic parameters $\mathrm{Pn}(\mathbf{A}), \mathrm{Gs}(\mathbf{B})$, and $\mathrm{Ci}(\mathbf{C})$ in transgenic and wild-type Arabidopsis thaliana plants five days after salt treatment.

\section{Enhanced expression of stress responsive genes in transgenic Arabidopsis plants}

The expression of $R D 22, R D 29 A, R A B 18, C O R 47$, and $A B A 1$, which are all stress responsive genes, was strongly enhanced in the overexpressed ChiMYB transgenic plants (Figure 9A-H) as compared to the non-transgenic plants treated with $250 \mathrm{mM} \mathrm{NaCl}$. When salt stress was not applied, the expression of these genes was quite low, and no significant differences were detected between transgenic and non-transgenic plants. However, the expression of $A B I 1$ and $H A B 1$ in the overexpressed ChiMYB transgenic plants was lower than that observed in non-transgenic Col-0 plants (Figure 9F-G). Nevertheless, the expression of $R D 22, R D 29 A, R A B 18, C O R 47$, and $A B A$ in transgenic plants was significantly enhanced compared to non-transgenic plants, and $C h i M Y B$ expression gradually increased. 


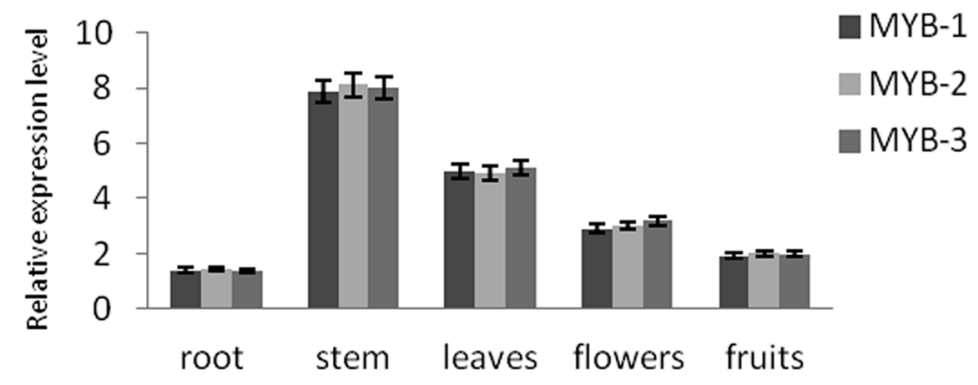

Figure 8. ChiMYB gene expression patterns in different organs under natural conditions.

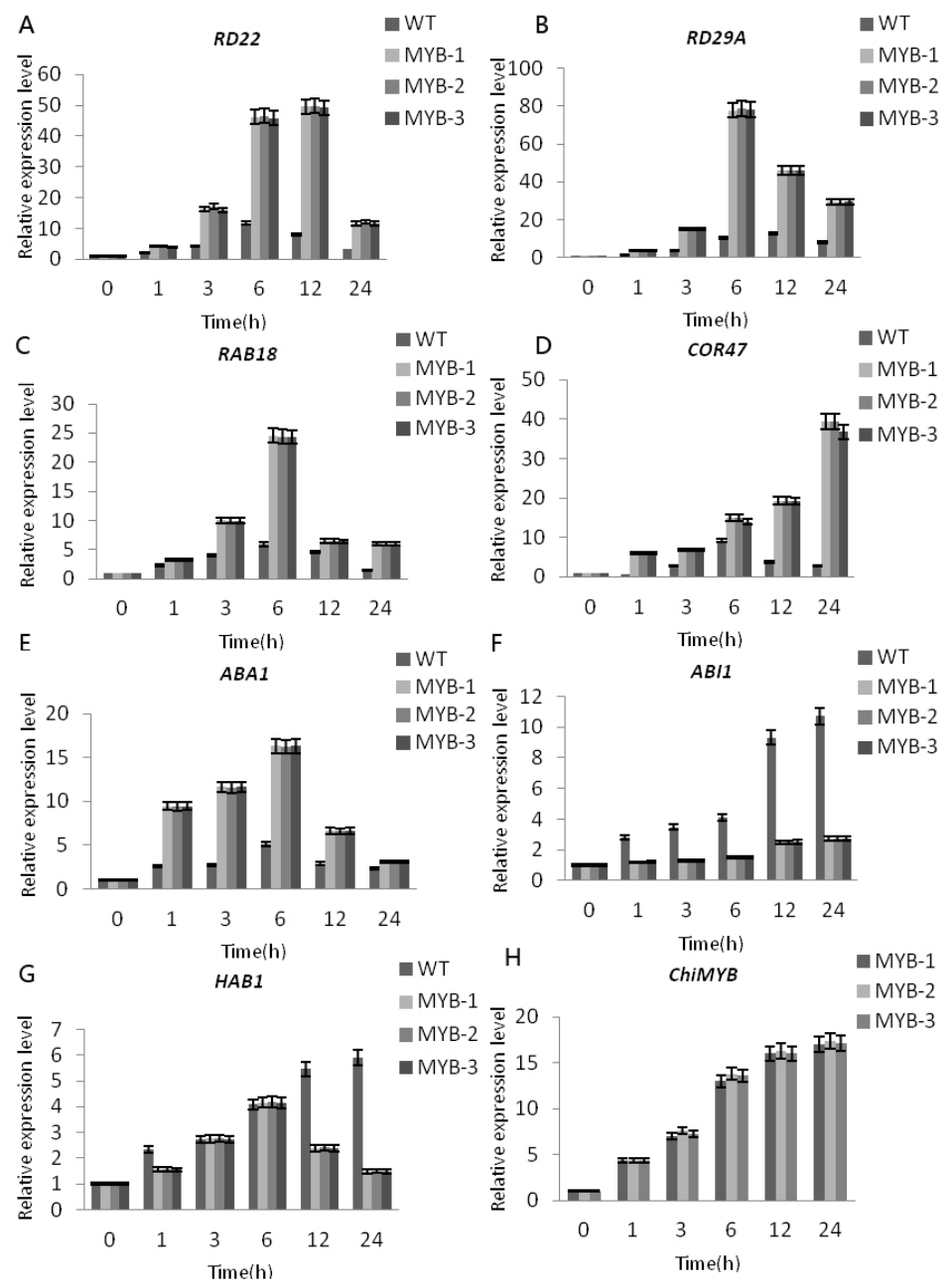

Figure 9. The expression of stress-responsive genes and ChiMYB in transgenic and non-transgenic Arabidopsis plants.

Genetics and Molecular Research 15 (3): gmr.15038985 


\section{DISCUSSION}

\section{ChiMYB transcription factor can improve plant tolerance to salt stress}

MYB transcription factors are essential for plant defense responses to biotic and abiotic stress. In this study, ChiMYB from $C$. indicum was isolated and expressed in Arabidopsis to characterize its function with regard to salt stress tolerance. Our results revealed that under salt stress, the survival rate of non-transgenic plants was approximately $30 \%$, while the transgenic plants exhibited normal growth and higher survival rates. Therefore, our data demonstrated that ChiMYB expression increased Arabidopsis plant tolerance to high salt concentrations. It is interesting to note that when under salt stress, the germination rate of the transgenic lines was slightly higher than that of non-transgenic control plants. These data suggest that ChiMYB-induced tolerance to salt stress might be associated with specific plant developmental stages. However, this hypothesis must be investigated further.

\section{ChiMYB transcription factor can increase plant chlorophyll content and photosynthetic efficiency}

The $\mathrm{chl} / \mathrm{chl} b$ ratio is associated with the degree of chloroplast thylakoid stacking. Therefore, when the chla/chlb value is low, the level of thylakoid stacking might be low, which consequently leads to poor thylakoid membrane stability. Ultimately, the light energy distribution between photosystems PSI and PSII will be negatively affected, and photosynthetic activity will decrease. The $\mathrm{Car} / \mathrm{Chl}$ value is related to plant stress tolerance and photosynthetic activity (Liu and Liu, 1991). Our data revealed that the chla/chl $b$ values of both transgenic and non-transgenic plants declined quickly when subjected to high $\mathrm{NaCl}$ concentrations. When the $\mathrm{NaCl}$ concentration was below $150 \mathrm{mM}$, the chla/chlb ratio of transgenic Arabidopsis plants did not change (Figure 6A). However, when under high salt concentrations, the ratio of chla/chlb in non-transgenic plants reduced rapidly while that of transgenic plants did not changed significantly, and this suggested that the transgenic plants were more tolerant to salt stress than non-transgenic control plants. As shown in Figure 6B, the Car/Chl ratio of the transgenic Arabidopsis plants increased gradually as the salt concentration increased, but the ratio decreased when subjected to a $150 \mathrm{mM}$ salt treatment. In contrast, the ratio of $\mathrm{Car} / \mathrm{Chl}$ of non-transgenic plants peaked at a concentration of $50 \mathrm{mM} \mathrm{NaCl}$, which was followed by a quick decline as the salt concentration increased. The change in the $\mathrm{Car} / \mathrm{Chl}$ ratio suggested that transgenic Arabidopsis plants were more tolerant to high salt concentrations than nontransgenic control plants. Therefore, our data indicate that ChiMYB improved the tolerance of plants to salinity.

The mechanisms underlying salt stress suppression of plant photosynthesis are not fully understood at this point. Under salt stress, the values of $\mathrm{Pn}, \mathrm{Gs}$, and $\mathrm{Ci}$ in the three transgenic Arabidopsis lines decreased, but the decrease was not as pronounced as that observed in the non-transgenic control. These results suggested that photosynthesis in transgenic Arabidopsis plants was not as strongly affected as that in the control plants. Furthermore, our results revealed that the ChiMYB transcription factor plays a positive regulatory role in improving photosynthesis. For instance, the stomatal factor might be critical to decreased photosynthetic capacity in A. thaliana (Xu and Chen, 1999).

Genetics and Molecular Research 15 (3): gmr.15038985 


\section{Subcellular localization of ChiMYB}

In this experiment, ChiMYB was fused to the GFP reporter gene under the control of a CaMV 35S promoter, and it was transiently expressed in onion epidermal cells. The ChiMYBGFP fusion protein was localized to the nucleus, indicating that ChiMYB targeted the nucleus as expected.

\section{ChiMYB regulates the expression of downstream genes involved in plant stress responses}

Several studies confirmed that MYB transcription factors are essential for signal transduction, regulation of downstream gene expression, and stress responses (Liu et al., 2001). Our data indicated that ChiMYB induced the expression of downstream genes in salt stress signaling pathways ( $R D 22, R D 29 A, R A B I 8, C O R 47, A B I 1, A B A 1$, and $H A B 1)$, which suggested that $C h i M Y B$-enhanced salt tolerance results from the induction of these resistance genes.

In summary, salinity is a serious problem that affects crop production, and the improvement and enhancement of transcription factor regulatory functions could be an effective approach for the improvement of crop stress tolerance. The application of transcription factors for this purpose has attracted a lot of attention. However, studies of MYB transcription factors mainly focus on single stress conditions, but additional efforts should focus on their regulatory functions and interactions between multiple signaling pathways. Further investigations of MYB transcription factor regulatory mechanisms that control multiple signaling pathway interactions will help elucidate plant responses to stress.

\section{Conflicts of interest}

The authors declare no conflict of interest.

\section{ACKNOWLEDGMENTS}

Research partially supported by the Fundamental Research Funds for the Central Universities (grant \#2572015EY03 awarded to Y.W. Zhou), by the State Key Laboratory of Tree Genetics and Breeding (Northeast Forestry University grant \#201105 awarded to F. Wang), and by the National Natural Science Foundation of China (grant \#31400590 awarded to M. He and grant \#31470698 awarded to Y.W. Zhou).

\section{REFERENCES}

Antonietta C and Paola S (2010). Cloning and molecular characterization of R2R3-MYB and bHLH- MYC transcription factors from Citrus sinensis. Tree Genet. Genomes 6: 101-112. http://dx.doi.org/10.1007/s11295-009-0232-y

Chen BJ, Wang Y, Hu YL, Wu Q, et al. (2005). Cloning and characterization of a drought-inducible MYB gene from Boea crassifolia. Plant Sci. 168: 493-500. http://dx.doi.org/10.1016/j.plantsci.2004.09.013

Clough SJ and Bent AF (1998). Floral dip: a simplified method for Agrobacterium-mediated transformation of Arabidopsis thaliana. Plant J. 16: 735-743. http://dx.doi.org/10.1046/j.1365-313x.1998.00343.x

Du H, Yang WJ, Liu L, Tang XF, et al. (2008). Cloning and functional identification of the Two MYB transcription factors GmMYBJ6 and GmMYBJ7 in Soybean. Acta Agron. Sin. 34: 1179-1187. http://dx.doi.org/10.1016/S1875$\underline{2780(08) 60042-5}$

Genetics and Molecular Research 15 (3): gmr.15038985 
Hoeren FU, Dolferus R, Wu Y, Peacock WJ, et al. (1998). Evidence for a role for AtMYB2 in the induction of the Arabidopsis alcohol dehydrogenase gene (ADH1) by low oxygen. Genetics 149: 479-490.

Hoshida H, Tanaka Y, Hibino T, Hayashi Y, etal. (2000). Enhanced tolerance to salt stress in transgenic rice that overexpresses chloroplast glutamine synthetase. Plant Mol. Biol. 43: 103-111. http://dx.doi.org/10.1023/A:1006408712416

Huang XS, Wang W, Zhang Q and Liu JH (2013). A basic helix-loop-helix transcription factor, PtrbHLH, of Poncirus trifoliata confers cold tolerance and modulates peroxidase-mediated scavenging of hydrogen peroxide. Plant Physiol. 162: 1178-1194. http://dx.doi.org/10.1104/pp.112.210740

Jiang YB, Wang C and Li DF (2008). Effect of oxalic acid on chlorophyll content and antioxidant system of alfalfa under high temperature stress. Pratacultural Sci. 25: 55-59.

Jin L (2002). Effect of salt-conercion on SOD isoenzymes of alfalfa's chloroplast. J. Qinghai Normal Univ. 2: 54-56.

Kirik V, Kölle K, Miséra S and Bäumlein H (1998). Two novel MYB homologues with changed expression in late embryogenesis-defective Arabidopsis mutants. Plant Mol. Biol. 37: 819-827. http://dx.doi. org/10.1023/A:1006011002499

Lakshmi A, Ramanjulu S, Veeranjaneyulu K and Sudhakar C (1996). Effect of $\mathrm{NaCl}$ on photosynthesis parameters in two cultivars of mulberry. Photosynthetica 32: 285-289.

Lea US, Slimestad R, Smedvig P and Lillo C (2007). Nitrogen deficiency enhances expression of specific MYB and bHLH transcription factors and accumulation of end products in the flavonoid pathway. Planta 225: 1245-1253. http:// dx.doi.org/10.1007/s00425-006-0414-x

Lichtenhaler HK and Wellburn AR (1983). Detemination of total carotenoids and chlorophyll a and b of leaf extracts in different solvent. Biochem. Soc. Trans. 603: 591-592. http://dx.doi.org/10.1042/bst0110591

Liu L, Du H, Tang XF, Wu YM, et al. (2008). The roles of MYB transcription factors on plant defense responses and its molecular mechanism. Yi Chuan 30: 1265-1271. http://dx.doi.org/10.3724/SP.J.1005.2008.01265

Liu Q, Zhang GY and Chen SY (2001). Structure and regulatory function of plant transcription factors. Chin. Sci. Bull. 45: $1465-1474$.

Liu ZY and Liu ZQ (1991). The prospect of genetics and breeding for photosynthesis in crops. J. Guizhou Agric. Coll. 10: 20-38.

Livak KJ and Schmittgen TD (2001). Analysis of relative gene expression data using real-time quantitative PCR and the $2^{-\Delta \Delta C t}$ method. Methods 25: 402-408. http://dx.doi.org/10.1006/meth.2001.1262

Luo J, Butelli E, Hill L, Parr A, et al. (2008). AtMYB12 regulates caffeoyl quinic acid and flavonol synthesis in tomato: expression in fruit results in very high levels of both types of polyphenol. Plant J. 56: 316-326. http://dx.doi. org/10.1111/j.1365-313X.2008.03597.X

Martin C and Paz-Ares J (1997). MYB transcription factors in plants. Trends Genet. 13: 67-73. http://dx.doi.org/10.1016/ $\underline{\text { S0168-9525(96)10049-4 }}$

Masaharu K, Shoji H, Naoki Y, et al. (2003). Cloning and characterization of cDNAs associated with the embryogenic dedifferentiation of tobacco immature pollen grains. Plant Sci. 164: 1057-1066. http://dx.doi.org/10.1016/S01689452(03)00111-0

Misra AN, Sahu SM, Misra P, et al. (1997). Sodium chloride induced changes in leaf growth,and pigment and protein contents in two rice cultivars. Biol. Plant. 39: 257-262. http://dx.doi.org/10.1023/A:1000357323205

Schwechheimer C, Zourelidou M and Bevan MW (1998). Plant transcription factor studies. Annu. Rev. Plant Physiol. Plant Mol. Biol. 49: 127-150. http://dx.doi.org/10.1146/annurev.arplant.49.1.127

Shan H, Chen S, Jiang J, Chen F, et al. (2012). Heterologous expression of the chrysanthemum R2R3-MYB transcription factor CmMYB2 enhances drought and salinity tolerance, increases hypersensitivity to ABA and delays flowering in Arabidopsis thaliana. Mol. Biotechnol. 51: 160-173. http://dx.doi.org/10.1007/s12033-011-9451-1

Shu S, Guo SR, Sun J, Yuan YH, et al. (2012a). Research Progress on Photosynthesis under Salt Stress. China Vegetables 18: 53-61.

Shu S, Yuan LY, Guo SR, et al. (2012b). Effects of exogenous spermidine on photosynthesis, xanthophyll cycle and endogenous polyamines in cucumber seedlings exposed to salinity. Afr. J. Biotechnol. 11: 6064-6074.

Singh K, Foley RC and Oñate-Sánchez L (2002). Transcription factors in plant defense and stress responses. Curr. Opin. Plant Biol. 5: 430-436. http://dx.doi.org/10.1016/S1369-5266(02)00289-3

Sudhir P and Sistla D (2004). Effects of salt stress on basic processes of photosynthesis. Photosynthetica 42: 481-486. http://dx.doi.org/10.1007/S11099-005-0001-6

Tang N and Chen XB (2014). Research of MYB transcription factors in plant and abiotic stress response. J. Biol. 31: 74-78.

Uimari A and Strommer J (1997). Myb26: a MYB-like protein of pea flowers with affinity for promoters of phenylpropanoid genes. Plant J. 12: 1273-1284. http://dx.doi.org/10.1046/j.1365-313x.1997.12061273.x

Genetics and Molecular Research 15 (3): gmr.15038985 
Vannini C, Locatelli F, Bracale M, Magnani E, et al. (2004). Overexpression of the rice Osmyb4 gene increases chilling and freezing tolerance of Arabidopsis thaliana plants. Plant J. 37: 115-127. http://dx.doi.org/10.1046/j.1365313X.2003.01938.x

Xu DQ and Chen YG (1999). Plant Physiology and Molecular Biology: The limiting factor of photosynthesis (Xu XW and Tang ZC, eds.). Science Press, Beijing, 262-276.

Yang WJ, Du M and Fang F (2008). Cloning and characterization of two new MYB transcription factor genes from soybean. Zhongguo Nong Ye Ke Xue 41: 961-970.

Genetics and Molecular Research 15 (3): gmr.15038985 